\title{
Identification of lines of electric lines of three-phase distribution networks in the composition of ASMAE
}

\author{
Turatbek Omorov ${ }^{1, *}$, Beishenaly Takyrbashev ${ }^{2}$, and Kubanychbek Zakiriaev ${ }^{1}$ \\ ${ }^{1}$ National Academy of Sciences, Bishkek, Kyrgyz Republic \\ ${ }^{2}$ PC "National electric network of Kyrgyzstan", Chui enterprise of high-voltage electrical networks, Bishkek, Kyrgyz Republic
}

\begin{abstract}
The problem of protection of a three-phase four-wire distribution network (DEN) with a voltage of $0.4 \mathrm{kV}$ from the interruption of electric power lines is considered. On the basis of the analysis of the values of complex resistances of interpersonal sections of the main line of the network, criteria are proposed that allow detecting critical situations associated with discontinuities in power transmission lines. Using these criteria makes it possible to localize the places of breaks of phase and neutral wires. Mathematical models and methods that are used to evaluate the complex resistances of interpersonal sections of a three-phase network are briefly considered. The obtained results are oriented for use in the automated system of electricity control and accounting (ASMAE).
\end{abstract}

\section{Introduction}

At present, automated systems for monitoring and accounting of electric power (ASMAE) are widely implemented in automation and informatization of power consumption processes in distribution electrical networks (DEN) with a voltage of $0.4 \mathrm{kV}$ [1-4]. The main structural and functional elements of these systems are data concentrators (DC), a set of electricity meters (EM) installed at the subscribers of the network. For the exchange of technological and service information between the subsystems of the ASMAE in the DC and EM there are telecommunication modules (TCM) built using modern data transmission technologies (GSM, Zigbee, PLC, etc.). The main functions of the DC: operational data collection from a group of electricity meters (EM); storage of received data; digital processing of information to solve relevant functional problems; information exchange with the upper level of management. In general, it should be noted, that in the framework of ASMAE, the tasks of commercial metering of electricity are being solved. At the same time, in order to increase the efficiency and intellectual level of automated systems, it is advisable to solve such important functional tasks as operational diagnostics of states [5-7] and optimization of operation modes of DEN [8-10]. Among the diagnostic tasks, it is especially important to identify the problem of identification and localization of breakage places of electric lines of a three-phase distribution network. To date, a number of approaches and tools for its solution have been developed [7].

The paper proposes one of the possible methods for solving it as part of ASMAE using real-time data from electricity meters installed at the network subscribers.

\section{Method of solution}

Three-phase DEN with a voltage of $0.4 \mathrm{kV}$ is considered, the design scheme of which is shown in the fig.1. For convenience in the scheme, the phases A, B, C are numbered respectively through the index variable $(k=\overline{1,3})$. The remaining notations have the following meaning: $E_{A}, E_{B}, E_{C}-$ instantaneous phase EMF; $Z_{v k}$ is the designation of the $v$ - th load (electric receiver) $(v=\overline{1, n})$, connected to the phase with the number $k ; I_{v k}, U_{v k}$ - instantaneous current and voltage on the load $Z_{v k} ; l_{v k}, z_{v k}$ - instantaneous current and resistance of the $v$-th interpersonal section (IS) of the $k$ th phase; $u_{v k}, u_{v}$ - instantaneous voltages respectively on the $v$ - th IS of the $k$-th phase and the neutral wire; $J_{v}, z_{v^{-}}$instantaneous current and resistance of the $v$-th section of the neutral wire; $U_{0 k}, l_{1 k}$ - are instantaneous voltages and currents, respectively, at the inputs of the respective phases.

Further, it is assumed that the following conditions are satisfied:

1) DEN operates in an asymmetric mode;

2) the phase and neutral wires of the network can have different cross sections, i.e. the resistances $z_{v k} \neq z_{v}$ $(k=\overline{1,3}, v=1, n)$, which are a priori unknown;

3 ) the system uses technical means to suppress the higher harmonic components of currents and voltages in the network;

\footnotetext{
Corresponding author: omorovtt@mail.ru
} 


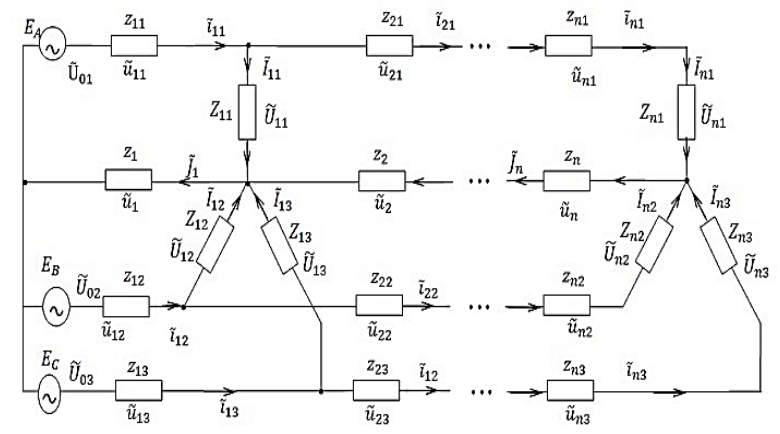

Fig.1. The design scheme of a three-phase DEN.

4) from electricity meters $\left(E m_{v k}\right)$ to the concentrator (DC) via communication channels at discrete instants $t \in\left[t_{\xi}, t_{\xi+1}\right]$ with a sampling rate $\Delta t_{\xi}=t_{\xi+1}-t_{\xi}$ $(\xi=1,2, \ldots)$ the following data arrive:

- current values of currents $I_{v k}$ and voltages $U_{v k}$ on loads $Z_{v k}$;

- power factors $\cos \varphi_{v k}$, o determined by the phase shifts $\varphi_{\nu k}$ between the corresponding voltages $U_{v k}$ and currents $I_{v k}$.

Note that the electrical state of the network at time $t \in\left[t_{\xi}, t_{\xi+1}\right]$ is described by the following vectors:

$I_{k}=I_{1 k}, I_{2 k}, \ldots, I_{n k}, \quad U_{k}=U_{1 k}, U_{2 k}, \ldots, U_{n k}$,

$u_{k}^{\prime}=u_{1 k}, u_{2 k}, \ldots, u_{n k}, i_{k}=l_{1 k}, l_{2 k}, \ldots, l_{n k}$,

$u=u_{1}, u_{2}, \ldots, u_{n}, \quad J=J_{1}, J_{2}, \ldots, J_{n}$,

$Z_{k}=z_{1 k}, z_{2 k}, \ldots, z_{n k}, \quad Z_{N}=z_{1}, z_{2}, \ldots, z_{n}, k=\overline{1,3}$,

where $I_{v k}, U_{v k}, u_{v k}, l_{v k}, u_{v}, J_{v}-$ are complex representations of the corresponding electrical variables indicated on the three-phase DEN circuit. The task is as follows:

1) define the vectors $I_{k}, U_{k}, u_{k}^{\prime}, l_{k}, u, J, Z_{k}, Z_{N}$, describing the electrical state of the network;

2) to identify the coordinates of the breaks of the phase and neutral wires of DEN.

Under the condition that the DEN operates in the normal (state) mode, the of determining the problem of the network is solved, in particular, the interpersonal complex currents $l_{v k}$ for $k$-th phase, as well as the resistance values $z_{v}^{*}$ of neutral wire segments. Further on the basis of these data we make the following vectors:

$$
i_{k}^{*}=i_{1 k}^{*}, i_{2 k}^{*}, \ldots, i_{n k}^{*}, \quad Z_{N}^{*}=\left[z_{1}^{*}, z_{2}^{*}, \ldots, z_{n}^{*}\right] .
$$

We note that the values of the base resistances $z_{v}^{*}$, $v=1, n$, very slowly depending on the current climatic conditions and for a sufficiently long period of time remain practically constant, i.e $z_{v}^{*}=$ const.

As is known, there are two types of breaks in power electric lines in the DEN:

1) breaks of phase lines;

2) a neutral wire breaks.

In the first case, when the wire of the $q$-th section of the $k$-th phase is broken, the vector $i_{k}$ has the following form:

$$
i_{k}=i_{1 k}, i_{2 k}, \ldots i_{q-1, k}, 0,0, \ldots, 0 .
$$

In the second case, if the wire of the $\eta$-th section of the neutral wire is broken, the vector $Z_{N}$ has the following form:

$$
Z_{N}=\left[z_{1}^{*}, z_{2}^{*}, \ldots, z_{\eta-1}^{*}, z_{\eta}, z_{\eta+1}^{*}, \ldots, z_{n}^{*}\right] .
$$

The analysis shows that in this case the following relations hold for the components of the vector $Z_{N}$ :

$$
\begin{gathered}
z_{\eta} \neq z_{\eta}^{*}, \\
z_{v}=z_{v}^{*}, \quad v=1,2, \ldots, \eta-1, \eta+1, \ldots, n,
\end{gathered}
$$

where $z_{\eta}$ - is the current resistance value determined for the considered discrete time moment $t$. Thus, second case, when condition (2) is satisfied, the vectors $Z_{N}^{*}$ и $Z_{N}$ are not equal, i.e.

$$
Z_{N} \neq Z_{N}^{*}
$$

Obviously, in the normal operation mode of the DEN (in the absence of line breaks), the condition $Z_{N}=Z_{N}^{*}$ is satisfied with a certain accuracy.

As a result, to localize breaks of phase and neutral network wires, the following criteria can be formulated:

- Criterion 1: when the wire of the $q$-th section of the $k$-th phase breaks off for the vector $l_{v k}$ the condition (1).

- Criterion 2: if the neutral wire $\eta$-th section is cut off, condition (3) is fulfilled.

It should be noted that in order to identify and localize breaks in electrical lines of DEN on the basis of the formulated criteria, it is necessary to solve the problem of determining the electrical state of a DEN. Because of its complexity, the use of known methods for calculating the three-phase network [11-14] presents certain difficulties. In this connection, in $[4,6,15]$, the methodological and algorithmic bases of estimating the variables and network parameters, oriented for application in the ASMAE structure, were proposed. The basis of this research area is the mathematical model [15], which allows currents and voltages on the network loads to be presented in a complex form:

$$
\begin{gathered}
I_{v k}=I_{v k}^{\mathrm{B}}+j I_{v k}^{\mathrm{M}}=I_{v k} e^{j \alpha_{v k}} \\
U_{v k}=U_{v k}^{\mathrm{B}}+j U_{v k}^{\mathrm{M}}=U_{v k} e^{j \psi_{v k}}, v=1, n, k=1,3,
\end{gathered}
$$

where the symbols "b" and " $\mathrm{m} "$ denote the real and imaginary parts of the corresponding complex variables; $I_{v k}, U_{v k}, \alpha_{v k}, \psi_{v k}-$ are the moduli (acting currents and voltages) of the corresponding complex variables and their phase shifts, respectively; $j=\overline{-1}-$ is an imaginary number.

The representations (4) and (5) make it possible to determine the complex currents $i_{v k}$ и $J_{v}$, occurring in the interpersonal sections of the network, as well as the corresponding voltages $u_{v k}$ и $u_{v}$. The corresponding models and algorithms are described in [4, 6, 15]. Knowledge of these data allows us to determine with some accuracy the estimates of the current values of the 
components of the vector parameters

$Z_{k}=z_{1 k}, z_{2 k}, \ldots, z_{n k}$ and $Z_{N}=z_{1}, z_{2}, \ldots, z_{n}$ of the three-phase network:

$$
\begin{array}{lc}
z_{v k}=u_{v k} \quad l_{v k}, & v=\overline{1, n}, \quad k=1,3, \\
z_{v}=u_{v} J_{v} & v=\overline{1, n} .
\end{array}
$$

\section{Conclusion}

Implementation of the above procedures (algorithms) for estimating the electrical state of the network is carried out in a data concentrator (DC) in real time.

The proposed models and computational algorithms for localizing faults of phase and neutral wires of a threephase distribution network based on the formulated criteria are oriented to create special software for the corresponding subsystem within the ASMAE system.

\section{References}

1. A. Ozhegov. ASMAE systems (Kirov, VyatSU, 2006)

2. A. Sapronov, S. Kuzhekov, V. Tyniansky. Izv.vuzov. Electromechanics, 1, 55 (2004)

3. A. Soldatov, U. Evdokimov. Devices and systems. Management, control, diagnostics, 3, 1 (2017)

4. T. Omorov, B. Takyrbashev. Electricity, 11, 4 (2016)

5. A. Soldatov. Devices and systems. Management, control, diagnostics, 6, 1 (2016)

6. T. Omorov, B. Takyrbashev. Control. Diagnostics, 5 (2017)

7. A. Ershov, O. Filatov, A. Mlotok etc. Electric station, 5, 28 (2016)

8. V. Idelchik. Calculations and optimization of modes of electrical networks and systems (M. Energoatomizdat, 1988)

9. T. Omorov, B. Takyrbashev. Instruments and systems, Management, control, diagnostics, 6, 11 (2016)

10. F.D. Kosoukhov, N.V. Vasilev, A.O. Filippov Electrical engineering, 6, 8 (2014)

11. K. Demirchyan, L. Neiman, A. Korovkin. Theoretical bases of electrical engineering (Spb. Peter, 2009)

12. U. Zhelezko. Loss of electricity. Reactive power. The quality of electricity (M. EHAS, 2009)

13. A. Harutyunyan. Electricity,10, 55 (2015)

14. Kochergin, A. Kobelev, N. Khrebtov, P. Kitashin, K. Terekhov. Fractal simulation. 1, 5 (2013)

15. T. Omorov, B. Takyrbashev, R. Osmonova. Energetik, 4, 28 (2017) 